\title{
Multi-objective Optimization of Afghanistan Kabul City Distribution Network for Real-time Voltage Control and Power Loss Reduction
}

\author{
Ahmad Samim Noorzad*, Tomonobu Senjyu \\ Department of Electrical and Electronics Engineering, University of the Ryukyus, \\ Senbaru 1, Nishihara-cho, Nakagami, Japan
}

Copyright (C) 2017 by authors, all rights reserved. Authors agree that this article remains permanently open access under the terms of the Creative Commons Attribution License 4.0 International License

\begin{abstract}
Afghanistan Kabul City Distribution System (KDS) is functioning in adverse condition, and there is always programmed or un-programmed load shedding imposed by the Power Distribution Company. There are four indicators to be optimized for the sustainability of KDS, power loss reduction, stability, reliability, preservation and protection. In this paper, the optimization of power loss reduction and optimal voltage control of Kabul Distribution System (KDS) in the presence of Distributed Generation (DG) and Step-Voltage Regulator (SVR) connected to the network is proposed. In particular, Kabul city has plentiful sources of renewable energy such as Photovoltaic (PV) and wind power as a countermeasure for diminishing the losses of the system. The Multi-Objective Particle Swarm Optimization (MOPSO) based on two objective functions regarding hourly power loss reduction and voltage profile improvement of the distribution network is considered. The application of PV as DG in distribution system regarding their optimal number, size, and location justifies as loss reduction, reducing the entire grid peak demand and minimizing the congestion of the network. However, the optimized tap ratios and position of SVR are treats to enhance and stabilize the voltage prole and to control the violation of the system voltage according to load conditions. The 24 hours technical data of the practically functioning Kabul city Distribution System Junction Station II are used as a case study. Finally, the proposed algorithm has been simulated using MATLAB ${ }^{\circledR}$ software, and the results are presented.
\end{abstract}

Keywords Distribution System Optimization, Loss Reduction, Real-time Voltage Control, Distributed Generation, Multi-objective Particle Swarm Optimization

\section{Introduction}

Kabul City is growing rapidly not only regarding population but also regarding infrastructure and economic devel- opment. The increase in population and economic growth has led to the need for improved and expanded infrastructure within the city including roads, water supply, sewerage, social infrastructure facilities like markets, education, health centers, and electricity supply. Due to insufficient energy production, there is a regular occurrence of power interruptions in the city characterized by power rationing and load shedding being imposed by the Kabul Electricity Department [1]. The power outages are not only due to the need for more generation plants to produce electricity that meets the demand but also by the poor state of the power distribution system which has technical and economic difficulties. At the present $28 \%$ of the Afghan households are connected to the power supply systems, and the connecting rate within the provinces differs in a wide range from zero in a rural area near to $100 \%$ in urban regions [2]. After the conflict years and devastating power system, there had been undoubted progressing in the infrastructure that today $70 \%$ of the citizens of the capital (Kabul) are connected to the electricity grid, where it made the power system more complex, periodic electric dispatch and power shortages [3].

Along with these disruptions, many researches has been accomplished based on distribution system optimization, allocation of DGs and SVRs in the feeders [4,5,6]. However, in this paper, the optimal number, allocation, and sizing of DGs and SVRs tap ratio and position in a real distribution network based on the actual topology of the system are used. In the suggested method, the voltage is maximized under the permissible voltage range by SVR in the feeders, and also the system losses are deflated by the combination of the two. The rest of the paper is organized as follows:

In section 2 the distribution model, power flow results of the network and the problem formulation for optimization are introduced. A method for optimal number and allocation of DG and SVR are given in section 3. Section 4 exhibits the simulation results. Finally, the conclusion of the paper is summarized in section 5 . 


\section{Distribution System Model and Problem Formulation}

Kabul distribution system Junction Station II has the radial topology with 158 buses and 157 branches fed by North Substation as shown in Figure 1[7]. There are industrial, commercial and residential loads in the network and is one of the congested Junction Stations in the city. All the nodes are $P-Q$, and the capacity of the system is $88.9 M V A$ with a nominal medium voltage of $20 \mathrm{kv}$. In Figure $2 \mathrm{a}$, the daily average load demand for 158 bus system in terms of Megawatt has been demonstrated. In this figure, it can be seen that the daily load curve patterns are similar. Statistically Kabul city electric power consumption in increases during the winter season, where in this study the network under highest demand over the year has been considered and analyzed. Moreover, as shown in Figure 2b, the average peak demand during winter's month (20 February to 21 March) is $78.33 \mathrm{MW}$ and the peak hour of the system is at 7 o'clock in the evening.

The power flow analysis of the system based on the ForwardBackward Sweep Algorithm has already been executed, where the primary node was set as a slack bus on the Junction Station II. The steady-state analysis results in inferior hourly voltage profile, as shown in Figure 3. The figure demonstrates the actual 158 bus system as a base case voltage profile in this paper. From this figure, it is evident that the functionality of the network is bellowed the standards, where the minimum $0.83 p u$ voltage is measured at bus number 111 during peak hour (i.e. 7 o'clock). Moreover, the total 5.6 $\mathrm{MVA}$ power loss of the system are calculated and illustrated in Figure 4 .

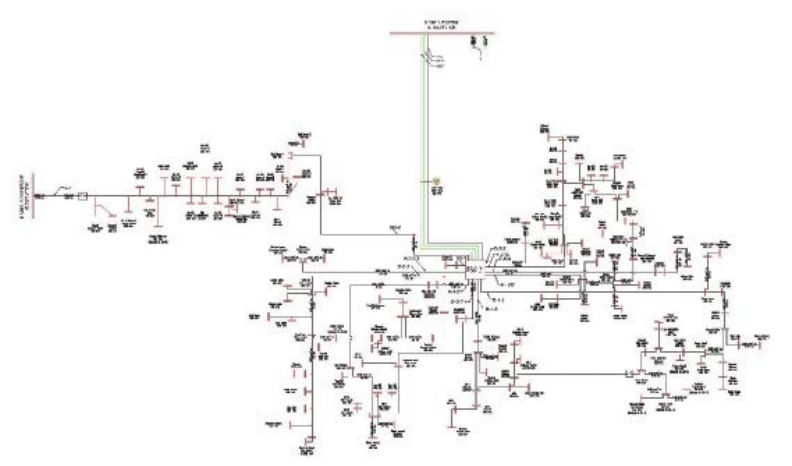

Figure 1. Kabul city Junction Station II distribution network

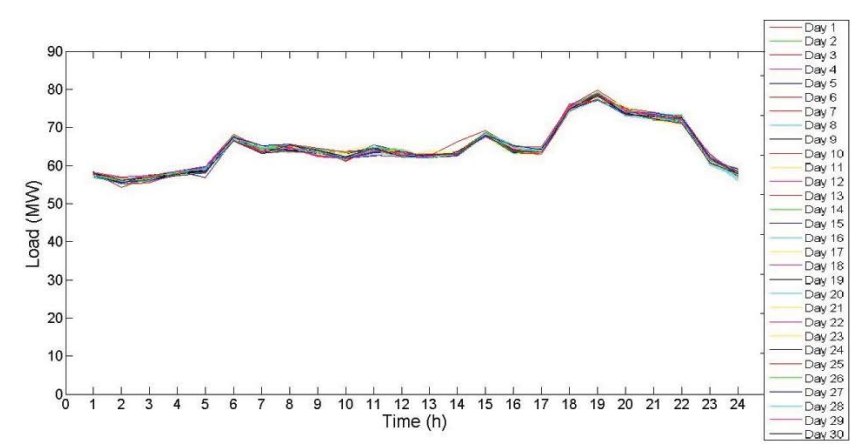

Figure 2a. Daily hourly load demand

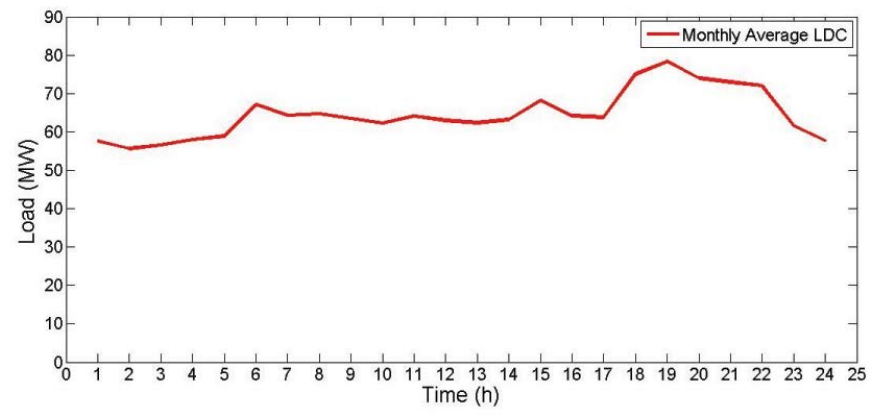

Figure 2b. Monthly hourly average load demand

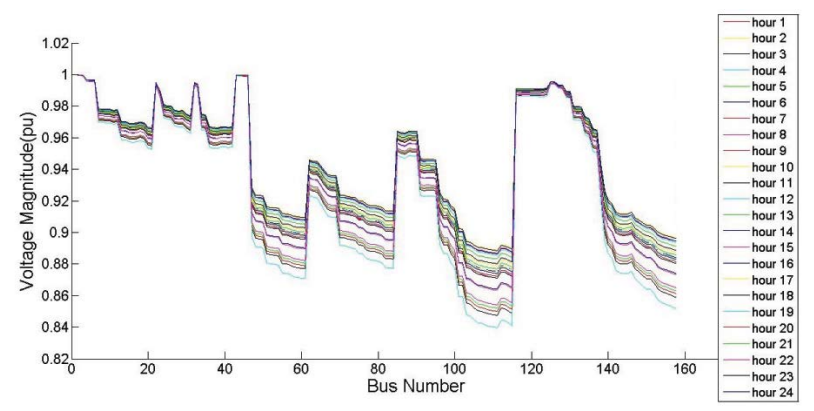

Figure 3. 158-bus system hourly voltage profile

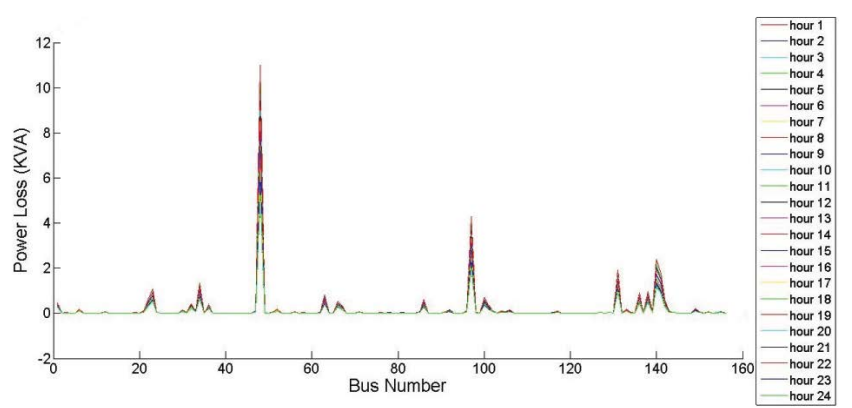

Figure 4. Hourly system power loss

\subsection{Objective Functions}

The losses in distribution systems mainly depend on its configuration, elements such as feeders and transformers parameters and also its operation state. Despite the fact, the efficiency of power dispatching defects by both active and reactive power loss of the system. As for the components model, the losses are mainly caused due to line impedances. Therefore, the recommendation for the system is to be highly analyzed, monitored and minimized [8]. Mathematically, the objective function of the problem is described as:

$$
O f_{1}=\sum_{t=1}^{24} \sum_{j=1}^{n-1} J_{j}^{2}\left(R_{j} \cos \varphi+j x_{j} \sin \varphi\right)
$$

where $t$ indicates for time in hour, $n$ is the total number of buses in the system, $I_{j}$ is the current flowing in the branch $j$, $R_{j}$ and $x_{j}$ are the branches resistance and reactance respectively. 
The objective function of time variant distribution voltage variation is shown in equation (2).

$$
O f_{2}=\sum_{t=1}^{24} \sum_{i=1}^{n}\left|\left(V_{n o m}-V_{i t}\right)\right|
$$

where $V_{\text {nom }}$ is the nominal voltage of the slack bus $(1.0 \mathrm{pu})$, $V_{i t}$ is the secondary $i$ bus voltage at hour $t$.

\subsection{SVR Model}

The Step-Voltage Regulator (SVR) in the medium voltage distribution system are used in the feeders for the prevention of voltage from sagging and swelling and maximize the voltage margin. The tap position and ratio are the key factors of SVR for controlling the network voltage and given in equation (3) and (4).

$$
\begin{gathered}
N_{S V R}=20 \frac{\left(95+S_{S V R}\left(\operatorname{Tap}_{S V R}-1\right)\right)}{100} \\
V_{2, S V R}=N_{S V R} * V_{1, S V R}
\end{gathered}
$$

where $N_{S V R}$ is the transformer ratio of SVR, $S_{S V R}$ describes the step size of the SVR ratio; $\operatorname{Tap}_{S V R}$ indicates the tap position, $V_{2, S V R}$ and $V_{1, S V R}$ correspondingly are the secondary and primary voltage of SVR.

In this study, the SVR is considered to have $1.25 \%$ step size with nine tap positions as shown in Table I.

Table 1. The relationship between tap position and tap ratio of SVR

\begin{tabular}{|c|c|}
\hline SVR Tap Position & SVR Tap Ratio \\
\hline 1 & 95 \\
\hline 2 & 96.25 \\
\hline 3 & 97.5 \\
\hline 4 & 98.75 \\
\hline 5 & 100 \\
\hline 6 & 101.25 \\
\hline 7 & 102.5 \\
\hline 8 & 103.75 \\
\hline 9 & 105 \\
\hline
\end{tabular}

Hence, the multi-objective function regarding minimization of the cumulative power loss of the distribution system and the deviation of the bus voltage from the indicated value (1.0pu) can be clarified from equation (1) and (2) bellow:

$$
\operatorname{minF}=W_{1} O f_{1}+W_{2} O f_{2}
$$

where $W_{1}+W_{2}=1$ and shows the weight for power loss reduction $\left(W_{1}\right)$ and voltage improvement $\left(W_{2}\right)$, the weighted values are either 0.5 .
The following operational constraints involve the DGs real and reactive power limits along with the limits of each bus voltage and upper and lower bound of SVR tap position.

$$
\begin{gathered}
P_{\min } \leq P \leq P_{\max } \\
Q_{\min } \leq Q \leq Q_{\max } \\
V_{\min } \leq V \leq V_{\max } \\
T a p_{\min } \leq \text { Tap } \leq \operatorname{Tap}_{\max }
\end{gathered}
$$

\section{Method of Optimal Allocation and Number of DG and SVR}

The proposed method Particle Swarm Optimization (PSO) is a population-based algorithm and its ties to birds flocking searching for food in the search space. In execution of PSO algorithm to optimize a problem, the swarm is acting like an accumulation of particles which will fly through the search space toward the optimum solution[9,10]. The mechanism of the particles movement adopted using its velocity and position equations. The velocity of the particle will be updated by the velocity equation, which in turn provides distance and direction to the particle and added to the particles current position, yields new particles position. The velocity and position updates of the particle are shown in equations (10) and (11) respectively.

$$
\begin{gathered}
V_{i, j,(k+1)}=W * V_{i, j,(k)}+C_{1} * r_{1}\left(P_{(b e s t), i, j}-P_{i, j,(k)}\right)+ \\
C_{2} * r_{2}\left(g_{(b e s t), j}-p_{i, j,(k)}\right)
\end{gathered}
$$

$$
P_{i, j,(k+1)}=P_{i, j,(k)}+V_{i, j,(k+1)}
$$

where $V_{i, j,(k)}$ is the velocity of particle $i$ for dimension $j$ at iteration $k, P_{i, j,(k)}$ denotes the particle $i$ position for dimension $j$ at iteration $k$, the subscript $(k+1)$ demonstrates the next iteration, $W$ is the inertial weight for controlling the velocities history on current velocity; the acceleration constants are $C_{1}$ and $C_{2} ; r_{1}$ and $r_{2}$ represents the random numbers within $[0,1]$ which are repeating in each iteration; $P_{(b e s t), i, j}$ symbolizes for the personal best position of particle $i$ for dimension $j$ and $g_{\text {(best }), j}$ denotes the best position discovered of all particles for dimension $j$.

Therefore, simultaneously several objective functions can be handled as a multi-objective optimization by taking weighted aggregation, and all the functions will be combined through a weighted combination as a single equation, as per equation (5).

\section{Simulation Results}

The simulation results reveal the MOPSO algorithm application by solving the multi-objective function of the 158-bus 
distribution network of Kabul city for overall system loss reduction and voltage control. The optimal six number of DGs and SVRs are determined located in various positions of the system. The optimal allocation of DGs are in bus number 10, 23, 84, 99, 111 and 148 as well as SVRs are optimally located in branches 9, 22, 84, 99, 111 and 149. Moreover, in Figure 5 the optimized 24-hour voltage profile for the network is illustrated. The application of MOPSO by the optimal installation of DG units and SVRs tap ratios has a tremendous effect on peak hour and also on the overall system voltage, where this figure clarifies a comprehensive improvement in bus voltages compare to the base case hourly voltages. Figure 6 is the minimum hourly system voltage magnitude where the minimal system voltage (i.e., power flow results) was $0.83 p u$ however by the optimization application the voltage has been raised to $0.865 p u$. The hourly DG penetration for optimal six number of generations is illustrated in Figure 7. The figure describes the DGs generation variation due to the demand variation of the network. The range of DGs apparent power production fluctuates between zero to $5 K V A$ within 24 hour simulation time. The SVR units tap ratios are operated within the range between $0.95 p u$ to $1.05 p u$ for 24-hour simulation as exhibited in Figures 8a to 8f. Figure 9a demonstrates the minimized power loss of the network and Figure $9 \mathrm{~b}$ showing the maximum hour based power loss of the network. In addition, the total power loss of the system (i.e., power flow results) was 5.6MVA although after simulation the loss reduced to $3.93 M V A$.

The simulation time was 10.5 hour, and the results clarify that the proposed method can adjust the voltage within a particular time, and also a considerable power loss reduction can acquire.

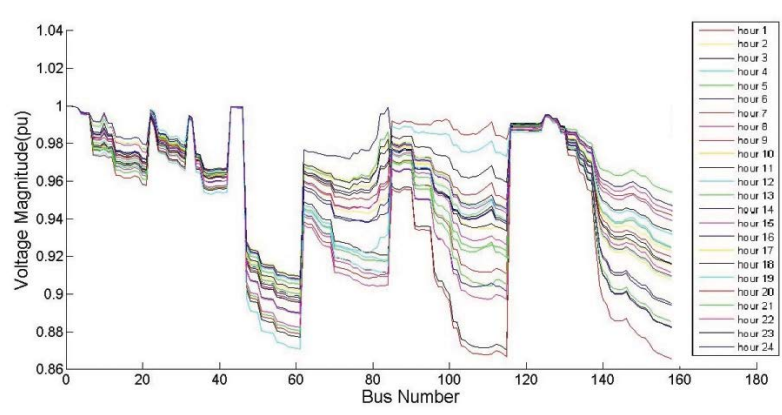

Figure 5. Optimized hourly system voltage profile

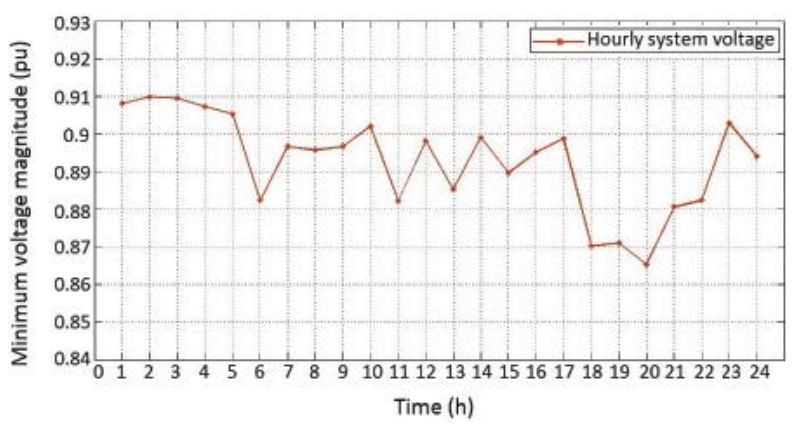

Figure 6. Minimum hourly voltage of the network

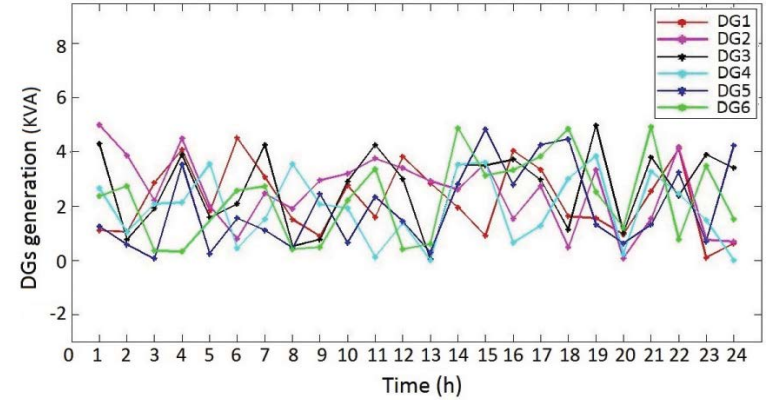

Figure 7. Hourly DGs generation

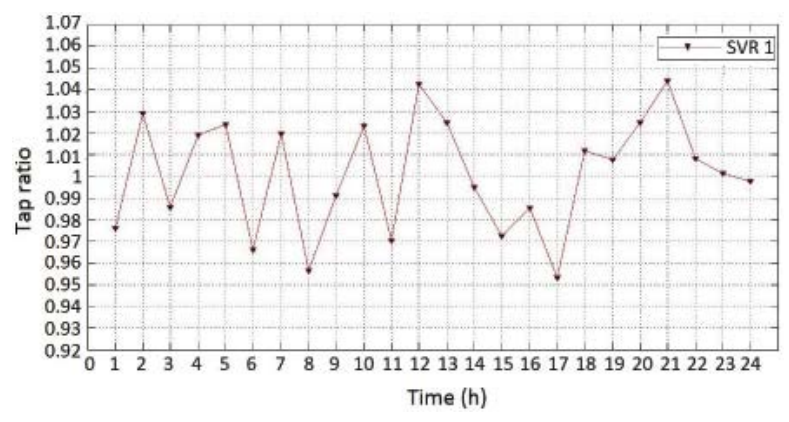

Figure 8a. SVR 1 hourly tap ratio

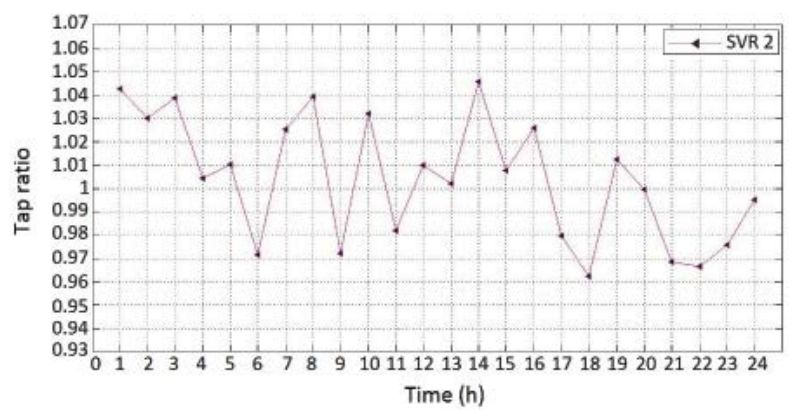

Figure 8b. SVR 2 hourly tap ratio

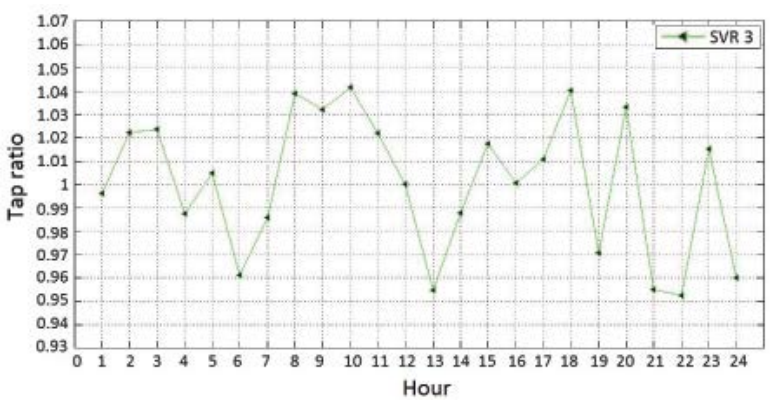

Figure 8c. SVR 3 hourly tap ratio

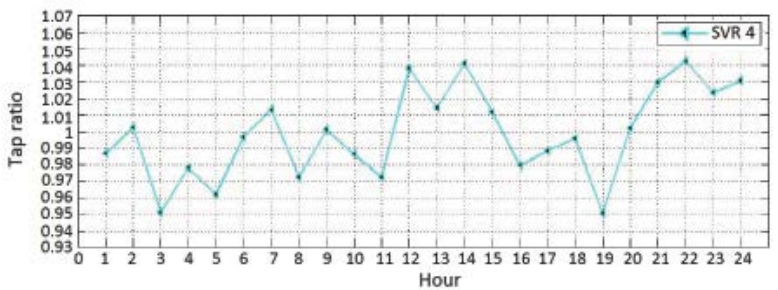

Figure 8d. SVR 4 hourly tap ratio 


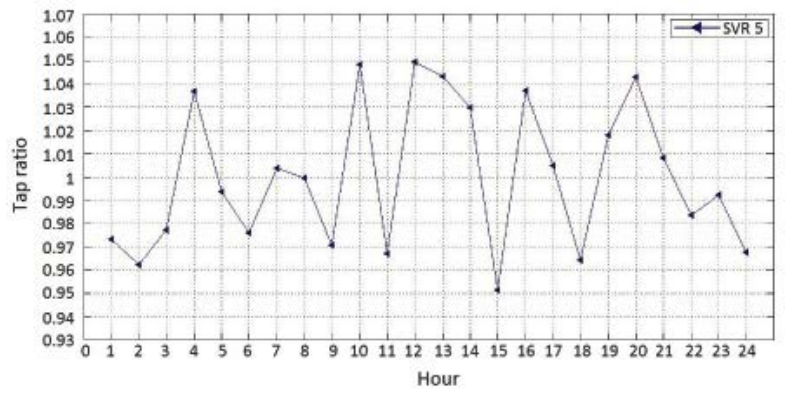

Figure 8e. SVR 5 hourly tap ratio

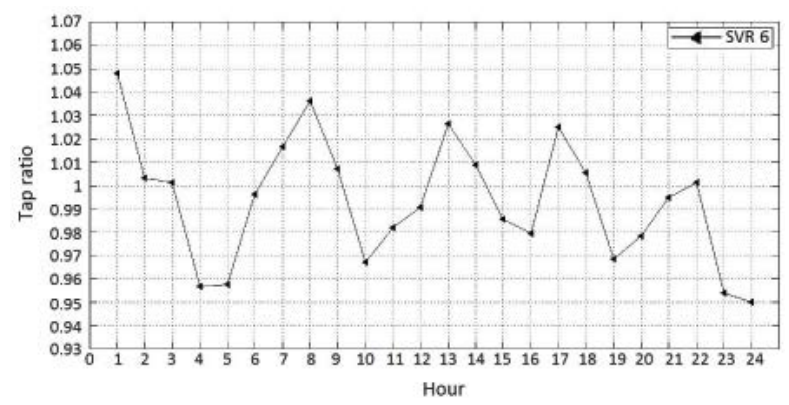

Figure 8f. SVR 6 hourly tap ratio

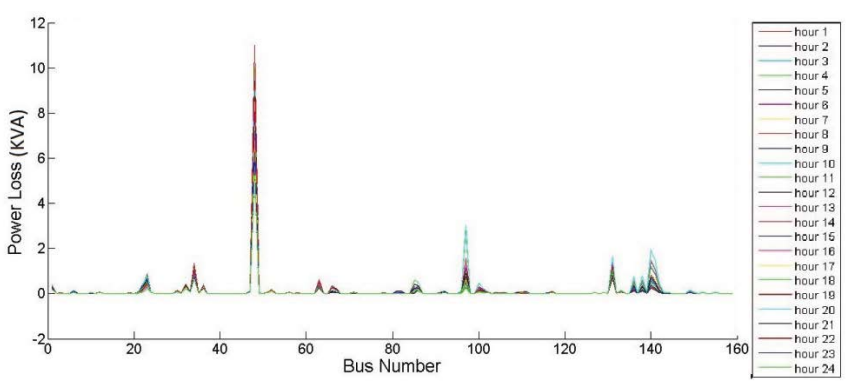

Figure 9a. Minimized power loss of the network

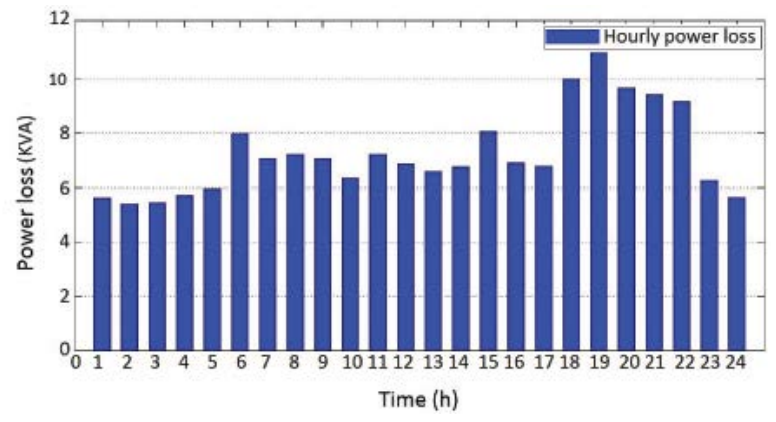

Figure 9b. Maximum hourly power loss

\section{Conclusion}

In this paper, a combination of DGs and SVRs for optimally controlling the voltage and diminishing the loss of a large scale real distribution network are proposed. As long as Kabul city have immense of renewable energy sources, especially the solar radiation, the photovoltaic generations are considered as DG for the penetration of real and reactive power to the system. The SVR devices which has a compact hardware, flexible tap changing capability and easy installation characteristic has been chosen for the regulation of voltage within short time intervals. The hourly system voltage is controlled by SVRs based on its optimal location and tap position using MOPSO algorithm. Finally, the simulation results affirmed the $30.6 \%$ loss reduction in the network and most of the bus voltage magnitudes had been boosted.

\section{Acknowledgements}

The author owe a debt of gratitude to Prof. Tomonobu SENJYU for his support and motivation, technical assistance of Ministry of Energy and Water (MEW), Da Afghanistan Breshna Sherkat (DABS); and financial aid of PEACE (Project for the Promotion and Enhancement of the Afghan Capacity for Effective Development)-JICA (Japan International Cooperation Agency).

\section{REFERENCES}

[1] Islamic Republic of Afghanistan, Power Sector Master Plan, Assistance Consultants Report, Project Number: 43497, May 2013.

[2] Mir Sayed Shah Danish, Toshihisa Funabashi. Explicit Recognition of Afghanistans Power Distribution Networks Problems and Technical Suggestions. TENCON 2014-2014 IEEE Region 10 Conference, Bangkok, pp. 1-6, 22-25 Oct.2014

[3] Islamic Republic of Afghanistan, Ministry of Economy, Inter-Ministerial Commission for Energy (ICE) Secretariat, Energy Sector Status Report, January-March, 2012.

[4] Tomonobu Senjyu, Yoshitaka Miyazato, Atsushi Yona, Naomitsu Urasaki, and Toshihisa Funabashi. Optimal Distribution Voltage Control and Coordination With Distributed Generation. IEEE transaction on Power Delivery, Vol. 23, No. 2, April 2008.

[5] Hiroyuki Mori and Takafumi Yoshida. Multi-objective Optimal Allocation of SVRs in Distribution Networks. IEEE 15th International Conference on Intelligent System Application to Power Systems, ISAP 09, p. 1-6, 8-12 Nov, 2009.

[6] Shuhei Takahashi, Yasuhiro Hayashi, Masaki Tsuji and Eiji Kamiya. Method of Optimal Allocation of SVR in Distribution Feeders with Renewable Energy Sources. Journal of International Council on Electrical Engineering, 2:2, 159-165. DOI: 10.5370/JICEE.2012.2.2.159. 
[7] Mohammad Nasir Ahmadi, Engineering and Planning General Manager, Da Afghanistan Breshna Sherkat (DABS). Kabul City Distribution System Survey. June, 2012. www.dabs.af.

[8] V. Tuzikova, Z. Muller, J. Svec, V. Valouch and J. Tlusty. Particle Swarm Based Optimization of Power Losses in Network Using STATCOM. International Conference on Renewable Energies and Power Quality (ICEPQ14), Cordoba (Spain), 8th to 10th April, 2014.
[9] Vipin Kumar and Sonajharia Minz. Multi-Objective Particle Swarm Optimization: An Introduction. Smart Computing Review, vol. 4, no. 5, October, 2014.

[10] Kennedy J and Eberhart RC. Particle Swarm Optimization. Proceedings of IEEE International Conference on Neural Networks, 1995. p. 1942-8. 